\title{
Design of an Integrated Air Coil for Current Sensing
}

\author{
Yoshikazu Kuwabara , Student Member, IEEE, Keiji Wada , Senior Member, IEEE, Jean-Michel Guichon , \\ Jean-Luc Schanen , Senior Member, IEEE, and James Roudet
}

\begin{abstract}
This article proposes a design procedure of an integrated air coil in a laminated bus bar for current sensing. The concept is based on an air coil inserted into the insulators inside the laminated bus bar, avoiding the use of intrusive current sensors with an additional stray inductance. First, the basic principle of the integrated current sensor is described, and the relationship between the mutual inductance and the structure of the air coil is discussed. Next, the frequency characteristic of the current sensor obtained based on an equivalent circuit is detailed, the parameters of which are derived from the sensor structure. A postprocessing method is proposed to achieve a 100-MHz frequency bandwidth. A prototype of the current sensor is designed based on the proposed design procedure, and the frequency characteristic is evaluated. The validations of the design procedure and the postprocessing method are confirmed experimentally.
\end{abstract}

Index Terms-Current sensor, frequency characteristic, laminated bus bar, postprocessing.

\section{INTRODUCTION}

W ITH the practical use of Silicon Carbide and Gallium Nitride, the recent power devices have improved dramatically. Such devices make the circuit possible to operate at a higher switching frequency compared with the use of conventional devices. This allows an increase of power density, by reducing the size of the passive elements [1], [2].

The stray inductance must be less than a few $\mathrm{nH}$ to keep an acceptable voltage surge on the wide bandgap (WBG) devices. A laminated bus bar between the dc capacitors and power devices is a technology used to realize a low stray inductance. A laminated bus bar is able to reduce the stray inductance by placing the plus and minus bus bars close together using thin insulators [3]. Many studies have discussed a laminated bus bar for some applications [4], [5] and have proposed an optimized structure of laminated bus bar with the value of stray inductance for the switching loss or surge

Yoshikazu Kuwabara and Keiji Wada are with the Department of Electrical and Electronic Engineering, Tokyo Metropolitan University, Tokyo 192-0397, Japan (e-mail: kj-wada@tmu.ac.jp).

Jean-Michel Guichon, Jean-Luc Schanen, and James Roudet are with the G2ELab, Univ. Grenoble Alpes, Grenoble Institute of Technology, 38031 Grenoble, France (e-mail: jean-luc.schanen@g2elab.grenoble-inp.fr). voltage [6], [7]. The value results in a rapid increase of the bus-bar current in case of a short circuit, and overcurrent protection should be implemented, needing current sensing devices [8]. These current sensing devices are also useful for device characterization (e.g., losses evaluation) [9].

Current sensors used in power converter circuits have been adapted to satisfy the different requirements in each application [10]. For instance, the current sensors for the characterization need accuracy and wide frequency bandwidth for measuring a current waveform. According to [10], the current sensors used for overcurrent protection require a short propagation time delay and high current sensor density $\left(\mathrm{A} / \mathrm{m}^{3}\right)$. Such current sensors require at least $100-\mathrm{MHz}$ bandwidth because the frequency components of the bus-bar current are up to dozens of MHz [11]-[13]. However, it is difficult to use conventional current sensors [14] in recent power converter circuits because the power circuit layout should be modified and stray inductance increases significantly, which is undesirable for WBG devices. The conventional current sensors are connected in the wire by clamping, inserting, or mounting. Therefore, those connections may require the modifications of circuit layout, and thus, increases the stray inductance with the insertion wire. Many sensors have been recently investigated to overcome this issue of conventional current sensors [15]-[23]. Some are featured by downsizing, while others have improved the structure for connection. Then, those sensors can be applied in a circuit without any increase in stray inductance. The current sensor based on the Rogowski coil in [19]-[23] can reduce the stray inductance. Those sensors focus on the current, which flows in the power device. However, there is no current sensor that is suitable for the laminated bus bar. The reason is that those Rogowski coil sensors that have been investigated so far are applied in each power device. However, there is a lack of consideration of the overall connection with the laminated bus bar. Consequently, this article discusses the current sensor that does not need any modification of the power converter circuit and is suitable for a laminated bus bar. An integrated air coil inside a laminated bus bar has been proposed [24], [25]. The integrated air coil is focused on the bus-bar current measurement, which is similar to the power device current. The basic concept of the integrated air coil was explained and validated in [24]. The current sensor detects a magnetic field, and current can be obtained from this measurement. Besides, the integrated air coil for the measurement of transient switching waveforms was discussed in [25]. 


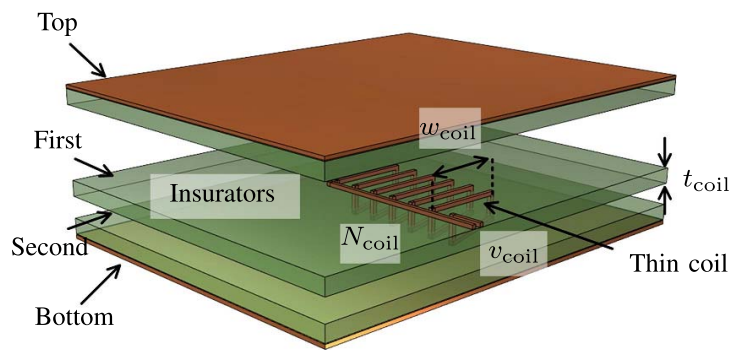

(a)

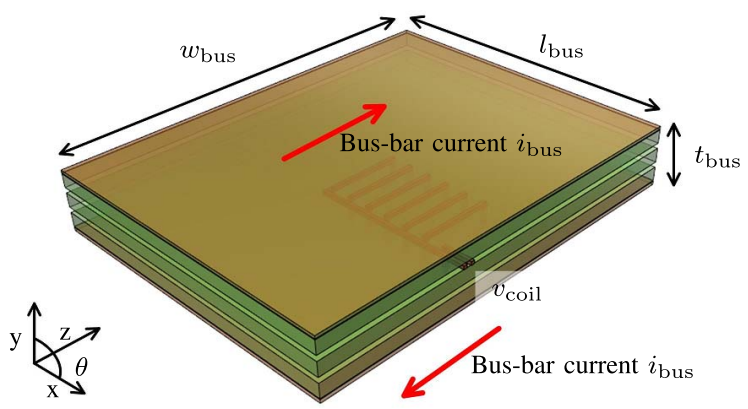

(b)

Fig. 1. Illustration of an integrated air coil. (a) Exploded view. (b) Implementation structure.

This article proposes a design procedure for an integrated air coil inserted inside a laminated bus bar, considering limitations imposed by the power circuit layout and the required bandwidth. Section II discusses the theoretical considerations of the current sensor. Section III describes the structure of a current sensor from a physical perspective. Section IV introduces a current sensor from an electrical circuit viewpoint using an equivalent circuit. The equation needed to determine the frequency bandwidth and the postprocessing method of improving the bandwidth are both revealed. Section V summaries the design procedure of the current sensor based on such discussions, and the current sensor is designed based on the proposed procedure. Experimental results with a current rated at $10 \mathrm{~A}$ are then utilized to validate the feasibility and effectiveness of the proposed method.

\section{Structure AND PRinciple of AN INTEGRATED AIR COIL}

\section{A. Structure of the Current Sensor}

Fig. 1 shows the structure of the integrated air coil for current sensing. Fig. 1(a) shows an exploded view of the current sensor, with the top and bottom layers being the plus and minus sides of the laminated bus bar. An air coil is placed inside the laminated bus bar between the top and bottom layers, and it is composed of two inner embedded layers. Consequently, the structure of the integrated air coil piles up such four layers. Fig. 1(b) shows the structural implementation of the current sensor. The top and bottom layers of the laminated bus bar are connected to the dc capacitors and power devices, and an air coil is inserted inside the laminated bus bar.

\section{B. Principle of the Current Sensor}

The equation of Ampere's law is represented as follows:

$$
\oint_{S} \mathbf{B}(\mathbf{t}) \cdot d \ell=\mu_{0} i_{S}
$$

where $\mu_{0}$ is the vacuum permeability. The magnetic flux density inside the coil is $B$, and the current $i_{S}$ flows through the area enclosed by curve $S$. The integrated air coil is similar to the Rogowski coil sensor because both current sensors use an air-core coil. The difference between both coils is related to the detection of different magnetic flux, which is generated by the current. The Rogowski coil sensor detects the magnetic flux, which is generated by a straight-wire current. And the integrated air coil detects the magnetic flux, which is generated by the loop-wire current because the laminated bus bar realizes a loop by the connection through dc capacitors and power devices.

To allow a simple theoretical analysis of an integrated air coil, the current distribution in the laminated bus bar is assumed to be uniform. Based on this assumption, an integrated air coil in (1) is represented by an analogy of the magnetic flux inside a solenoid coil as follows:

$$
B_{0} l_{\text {bus }}=\mu_{0} i_{\text {bus }}
$$

where $i_{\text {bus }}$ is the bus-bar current and the $l_{\text {bus }}$ is the length of the laminated bus bar. The magnetic flux $B_{0}$ in the laminated bus bar can be expressed as follows:

$$
B_{0}=\mu_{0} \frac{i_{\text {bus }}}{l_{\text {bus }}} \text {. }
$$

Therefore, the induced voltage of the air coil $v_{\text {coil }}$ is achieved when the bus-bar current $i_{\text {bus }}$ flows in a laminated bus bar, which can be represented by Faraday's law as follows:

$$
v_{\text {coil }}=-\frac{d \Phi_{\text {coil }}}{d t}=-S_{\mathrm{cc}} \frac{d B_{0}}{d t}=-\frac{\mu_{0} S_{\mathrm{cc}}}{l_{\text {bus }}} \frac{d i_{\text {bus }}}{d t}
$$

where $S_{\mathrm{cc}}$ is the total amount of cross section area in the air coil. Accordingly, the current sensor can measure only the ac component. An integrated air coil can obtain the bus-bar current $i_{\text {bus }}$ using the time integration of $v_{\text {coil }}$ as follows:

$$
i_{\text {bus }}(t)=\frac{l_{\text {bus }}}{\mu_{0} S_{\mathrm{cc}}} \int v_{\text {coil }}(t) d t .
$$

The mutual inductance $M$ of the integrated air coil can be defined through a uniform current distribution

$$
M=\mu_{0} \frac{S_{\mathrm{cc}}}{l_{\mathrm{bus}}} .
$$

In addition, the sensor has the noise immunity against the common mode. The reason is that the magnetic flux that occurred from the common mode is canceled in the laminated bus bar.

\section{Physical Discussion of An Integrated Air Coil}

\section{A. Design Parameters of the Mutual Inductance}

The mutual inductance $M$ is designed by the amount of cross section area in the air coil $S_{\mathrm{cc}}$, as shown in (6) because the length of the laminated bus bar $l_{\text {bus }}$ is fixed depending 


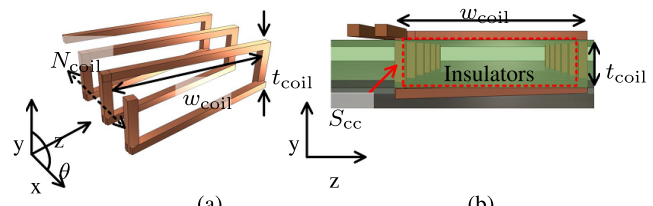

(a)

(b)

Fig. 2. Structure of the air coil. (a) Example of the air coil. (b) Cross-section area of the air coil.

TABLE I

PARAMETERS OF THE LAMINATEd Bus BAR

\begin{tabular}{l|l}
\hline Length $l_{\text {bus }}$ & $90 \mathrm{~mm}$ \\
\hline Width $w_{\text {bus }}$ & $115 \mathrm{~mm}$ \\
\hline Copper thickness $t_{\text {copper }}$ & $35 \mu \mathrm{m}$ \\
\hline
\end{tabular}

on the circuit structure. Fig. 2(a) shows an example of the air coil, and Fig. 2(b) shows the cross section area of the coil. The amount of cross section area $S_{\mathrm{cc}}$ can be described as follows:

$$
S_{\mathrm{cc}}=\left(w_{\text {coil }} t_{\text {coil }}\right) \times N_{\text {coil }}
$$

where $w_{\text {coil }}$ is the width of the coil, and $t_{\text {coil }}$ is the thickness of the coil. In addition, $N_{\text {coil }}$ represents the number of coil turns, and the $t_{\text {coil }}$ is dependent on the insulator design of the bus bar. Consequently, the sensitivity of the integrated air coil can be set by the structure.

\section{B. Current Density and Distribution in Laminated Bus Bar}

The current density and distribution in the laminated bus bar is a function of frequency to guarantee that the coefficient will not change too much with frequency when analyzing the mutual inductance value. In order to satisfy the theoretical assumption, the air coil should be placed in the location where the current distribution is uniform in the laminated bus bar. This evaluation is conducted to determine the location and estimating the mutual inductance. Therefore, the evaluation is conducted based on simulation.

The current density and distribution in the laminated bus bar can be evaluated using the simulation software based on a partial element equivalent circuit method [26]. The current density in the laminated bus bar for an integrated air coil was discussed in [24].

For the evaluation, the length $l_{\text {bus }}$ and width $w_{\text {bus }}$ of the laminated bus bar and the copper thickness $t_{\text {copper }}$ are set as shown in Table I. dc capacitors are connected in parallel in order to describe the realistic laminated bus bar condition. Fig. 3 shows the current density in the laminated bus bar at $1 \mathrm{~Hz}$ and $1 \mathrm{MHz}$. It is confirmed that the current density near the power devices is high and concentrated and that the density near the dc capacitors is shared by each capacitor. The part that has the same current density indicates a uniform location of the current distribution in the bus bar. Therefore, the current distribution can also be confirmed through Fig. 3 .

\section{Electrical Circuit Discussion of AN INTEGRATED AIR COIL}

\section{A. Equivalent Circuit of the Current Sensor}

The equivalent circuit of the current sensor should be represented, including the stray components, according to the range

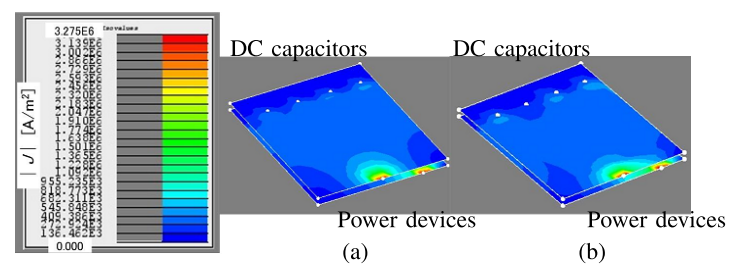

Fig. 3. Current density in a laminated bus bar. (a) $1 \mathrm{~Hz}$. (b) $1 \mathrm{MHz}$.

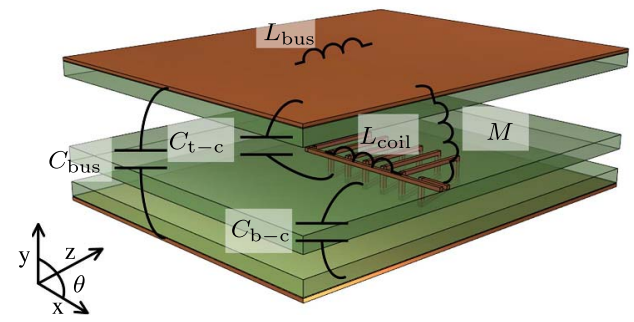

Fig. 4. Stray parameters of the integrated air coil.

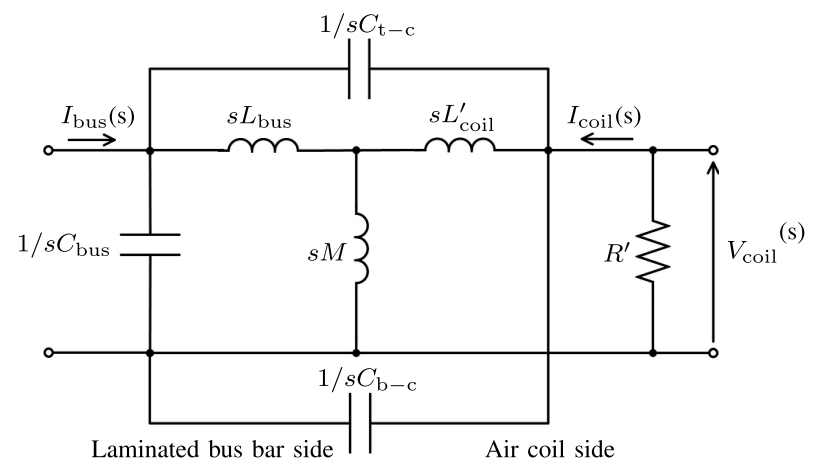

Fig. 5. Equivalent circuit of the current sensor with output resistance.

of the measurement frequency. The reason is that the stray parameters affect the frequency characteristic of the current sensor. Fig. 4 shows the stray components of the current sensor where $C_{t-c}$ and $C_{b-c}$ are the capacitance between the bus bar and the air coil wire, $C_{\text {bus }}$ is the bus-bar capacitance, $L_{\text {bus }}$ is the bus-bar self-inductance, and $L_{\text {coil }}$ is the air coil selfinductance. The line capacitance between the air coil wires is assumed to be zero because this capacitance can be expected to be much smaller than the other capacitances. Fig. 5 shows the equivalent circuit of the integrated air coil. For measuring the bus-bar current, the frequency range needs to be considered until $100 \mathrm{MHz}$ in the equivalent circuit because the busbar current includes high-frequency components until dozens of $\mathrm{MHz}$. In this range, the bus-bar capacitance $C_{\text {bus }}$ should be considered because the capacitance has a dominant value in the high-frequency region in the equivalent circuit. Fig. 6 shows the equivalent circuit of the integrated air coil until $100 \mathrm{MHz}$. Here, $L_{\text {coil }}^{\prime}$ is the air coil self-inductance, which reflects the number of coil turns $N_{\text {coil }}$ to the laminated bus bar side. The output resistance $R^{\prime}$ is connected for considering the air coil side impedance and $R^{\prime}$ also reflects the $N_{\text {coil }}$. The laminated bus bar side includes $C_{\text {bus }}, L_{\text {bus }}$, and $M$, and the air coil side includes $L_{\text {coil }}^{\prime}, M$, and $R^{\prime}$. 


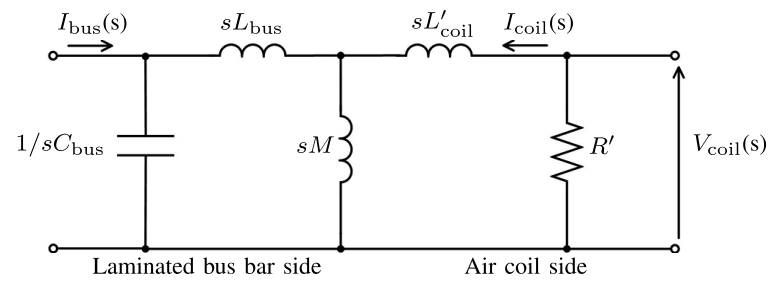

Fig. 6. Equivalent circuit of the current sensor with output resistance until $100 \mathrm{MHz}$.

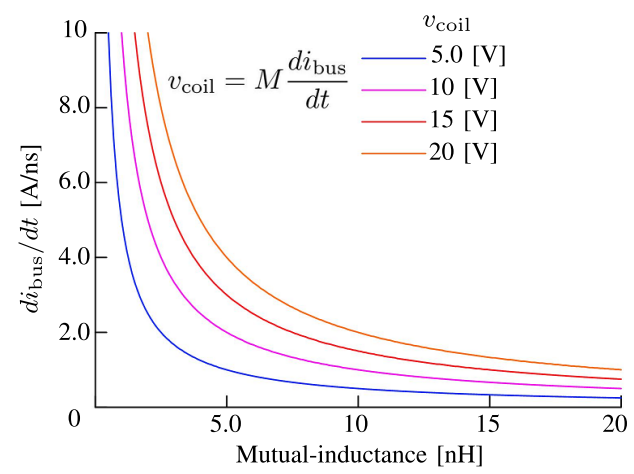

Fig. 7. Relationship between mutual inductance $M$ and $d i_{\text {bus }} / d t$.

\section{B. Designable Stray Parameters of the Current Sensor}

When the structure of the current sensor is determined, the stray parameters that remain to be designed are the mutual inductance $M$ and the air coil self-inductance $L_{\text {coil }}^{\prime}$. However, the $C_{\text {bus }}$ and $L_{\text {bus }}$ depend on the structure of the laminated bus bar.

1) Mutual Inductance $M$ : The induced voltage $V_{\text {coil }}(s)$ is represented by the bus-bar current $I_{\text {bus }}(s)$ and the air-coil current $I_{\text {coil }}(s)$ as follows:

$$
V_{\text {coil }}(s)=s M I_{\text {bus }}(s)+s L_{\text {coil }}^{\prime} I_{\text {coil }}(s) .
$$

The $I_{\text {coil }}(s)$ is assumed to be much smaller than $I_{\text {bus }}(s)$ owing to the output resistance $R^{\prime}$. Therefore, $I_{\text {coil }}(s)$ is assumed to be zero.

In the time domain, the induced voltage $v_{\text {coil }}$ is generated due to $d i_{\text {bus }} / d t$. The mutual inductance $M$ needs to be set by the relation between the $d i_{\text {bus }} / d t$ and the desired value of the induced voltage $v_{\text {coil }}$, according to signal detection and processing of the induced voltage. Fig. 7 shows the analysis results of such a relationship, and the mutual inductance can be set.

2) Air Coil Inductance $L_{\text {coil }}^{\prime}$ : The self-inductance $L_{\text {coil }}^{\prime}$ depends on $N_{\text {coil }}$. The turn ratio is determined through the inverse of the number of coil turns because the number of laminated bus-bar turns is one turn. The turn ratio squared $\left(1 / N_{\text {coil }}^{2}\right)$ is multiplied to the air coil self-inductance. The number of coil turns depends on the uniform location of the current distribution in the laminated bus bar because the air coil needs to be placed in this location. The self-inductance $L_{\text {coil }}^{\prime}$ and the output resistance $R^{\prime}$ which are in the air coil side must not modify the bus-bar current. In other words, the impedance of $L_{\text {coil }}^{\prime}$ and $R^{\prime}$ has to be much larger than the impedance of $M$ carrying the bus-bar current. This leads to the following equation:

$$
\omega M<\sqrt{\left(\omega L_{\text {coil }}^{\prime}\right)^{2}+\left(R^{\prime}\right)^{2}} .
$$

\section{Postprocessing Method for Extending the Bandwidth}

All stray parameters in Fig. 6 need to be considered in the postprocessing to achieve a $100-\mathrm{MHz}$ bandwidth. The transfer function $G(s)$ is defined by the bus-bar current $I_{\text {bus }}(s)$ and the induced voltage of the air coil $V_{\text {coil }}(s)$ as follows:

$$
\begin{aligned}
G(s) & =\frac{V_{\text {coil }}(s)}{I_{\text {bus }}(s)} \\
& =\frac{s}{K_{1} s^{3}+K_{2} s^{2}+K_{3} s+K_{4}}
\end{aligned}
$$

where the coefficients $\left(K_{1}-K_{4}\right)$ are determined based on the stray parameters of the current sensor shown in Fig. 6. Equation (11) takes all stray parameters of the current sensor into account. Equations (12)-(15) show each of the coefficients:

$$
\begin{aligned}
& K_{1}=\left\{C_{\text {bus }}\left(L_{\text {bus }} L_{\text {coil }}^{\prime}+L_{\text {bus }} M+L_{\text {coil }}^{\prime} M\right)\right\} / M R^{\prime} \\
& K_{2}=\left\{C_{\text {bus }}\left(L_{\text {bus }}+M\right)\right\} / M \\
& K_{3}=\left(L_{\text {coil }}^{\prime}+M\right) / M R^{\prime} \\
& K_{4}=1 / M .
\end{aligned}
$$

From (11), the bus-bar current can be represented as follows:

$$
I_{\text {bus }}(s)=\left(K_{1} s^{2}+K_{2} s+K_{3}+K_{4} / s\right) \times V_{\text {coil }}(s) .
$$

Therefore, the bus-bar current is shown in the time domain as follows:

$$
i_{\text {bus }}(t)=\left(K_{1} \frac{d^{2}}{d t^{2}}+K_{2} \frac{d}{d t}+K_{3}+K_{4} \int d t\right) \times v_{\text {coil }}(t) .
$$

Consequently, the frequency bandwidth of the integrated air coil can be extended using (17) in the numerical calculation of the computer. Then, the current sensor can accurately measure the switching waveforms.

\section{ExPERIMENTAL VALidation Using Prototype}

\section{A. Design Procedure of an Integrated Air Coil}

Fig. 8 shows the overall design procedure of an integrated air coil in a laminated bus bar.

Step 1: $\mathrm{A} d i_{\text {bus }} / d t$ is determined within a certain range based on the characteristic of the power devices. The mutual inductance of the current sensor is then set based on the $d i_{\text {bus }} / d t$ and the maximum limitation of an induced voltage $v_{\text {coil }}$.

Step 2: The cross section area of the bus-bar conductor is determined using the current value. The thickness of the insulators inside the laminated bus bar is determined by the application of a power converter. Therefore, $L_{\text {bus }}$ and $C_{\text {bus }}$ are dependent on the application.

Step 3: The current distribution in the laminated bus bar is analyzed to determine the uniform location, and an air coil is placed in that location. 


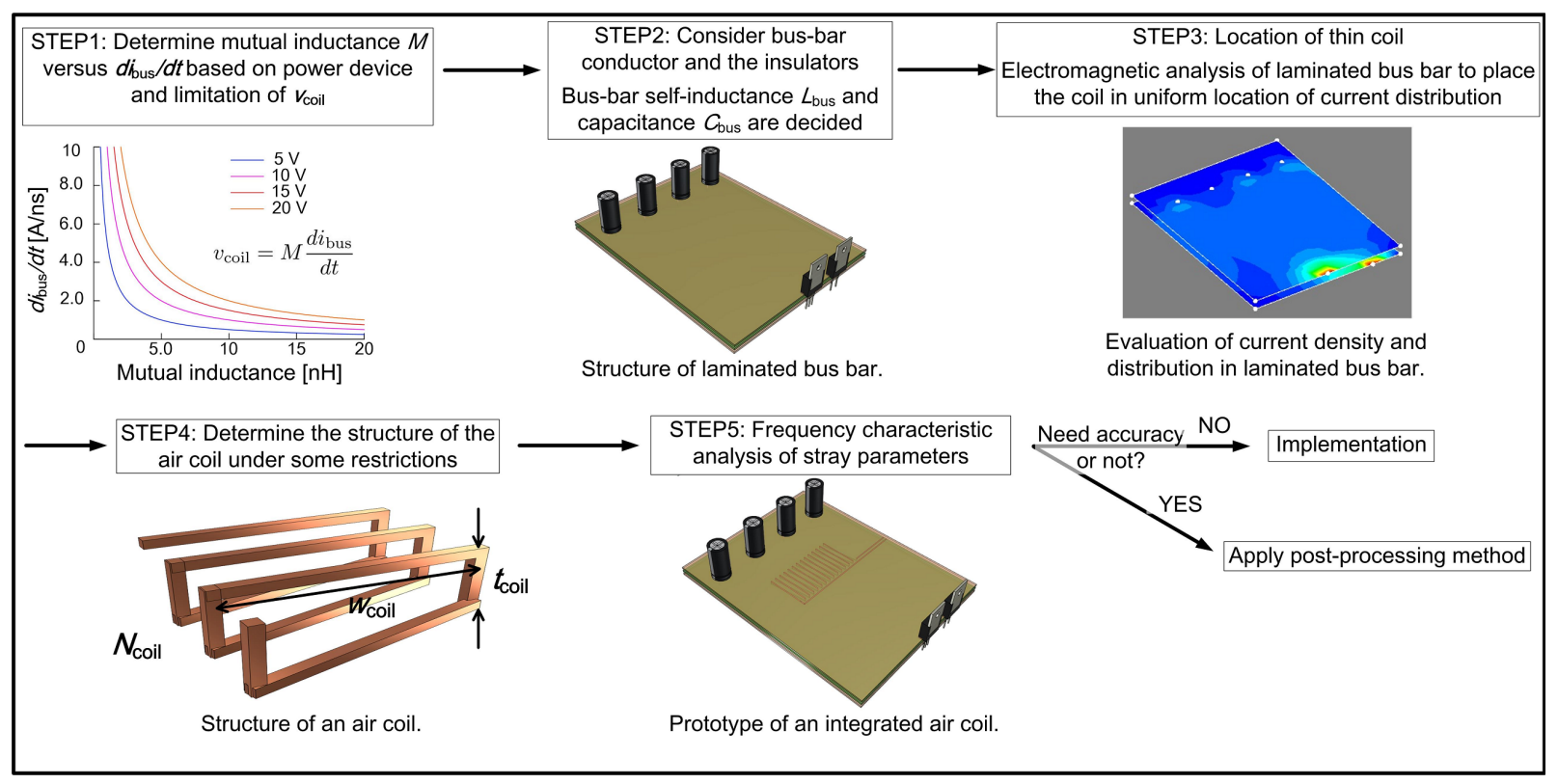

Fig. 8. Design procedure of an integrated air coil.

Step 4: The structure of the air coil is considered under the laminated bus bar structure and the value of the mutual inductance $M$.

Step 5: The frequency characteristic of the current sensor is evaluated by analyzing the stray parameters for each circuit. Whether a postprocessing method is necessary to obtain a frequency bandwidth of the $100 \mathrm{MHz}$ is then determined. If an accurate switching waveform needs to be measured by the current sensor, the postprocessing method is considered to improve the frequency bandwidth.

\section{B. Implementation of the Prototype}

The current sensor is designed based on Fig. 8 for the measurement of a switching waveform where $d i / d t$ is $1.5 \mathrm{~A} / \mathrm{ns}$. The induced voltage $v_{\text {coil }}$ is set to be less than $20 \mathrm{~V}$. Hence, according to Step 1, the mutual inductance $M$ is determined to be $13.3 \mathrm{nH}$ from Fig. 7. The dimensions of the laminated bus bar are designed for a current of $10 \mathrm{~A}$. The laminated bus bar is supposed to be used in the case dc capacitors are connected in parallel. Copper is used as the bus-bar conductor. According to Step 2, Table I shows the length $l_{\text {bus }}$ and the width $w_{\text {bus }}$ of the laminated bus bar, and the copper thickness $t_{\text {copper }}$ in the prototype. A $4.8-\mathrm{mm}$ thickness of the insulators inside the laminated bus bar is chosen. The thicknesses of the minus and plus bus bar layers and the thickness of the air coil layer $t_{\text {coil }}$ are restricted to $1.6 \mathrm{~mm}$.

The laminated bus bar is modeled in the simulation, and the current distribution is evaluated, as shown in Fig. 3. Then, the uniform location of the current distribution is near the connections of the dc capacitors in this prototype, and the air coil is placed in this location according to Step 3. According to Step 4 , the $w_{\text {coil }}$ is determined to be $30 \mathrm{~mm}$ in order to place the air coil in the location. Then, the $N_{\text {coil }}$ is determined to

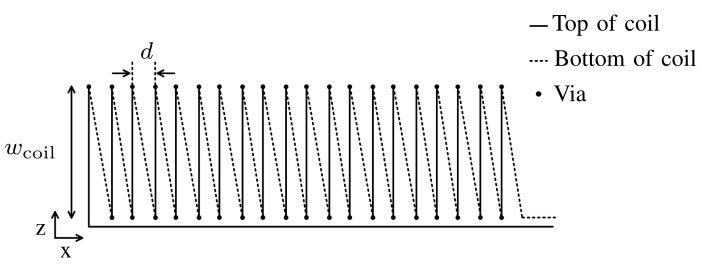

Fig. 9. Design of the air coil.

TABLE II

Air CoIl Parameters

\begin{tabular}{l|l}
\hline Thickness of the air coil $t_{\text {coil }}$ & $1.6 \mathrm{~mm}$ \\
\hline Sensor width $w_{\text {coil }}$ & $30 \mathrm{~mm}$ \\
\hline Number of turns $N_{\text {coil }}$ & $20 \mathrm{turns}$ \\
\hline Distance of each via $d$ & $3 \mathrm{~mm}$ \\
\hline
\end{tabular}

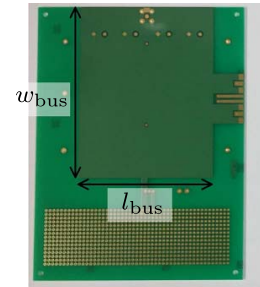

(a)

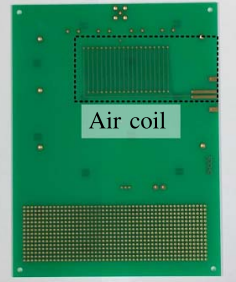

(b)

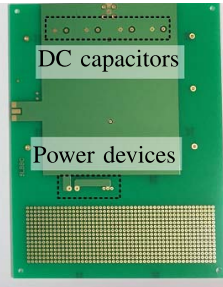

(c)
Fig. 10. Prototype of the integrated air coil. (a) Minus bus bar. (b) Air coil. (c) Plus bus bar.

be 20 to obtain a mutual inductance of $13.3 \mathrm{nH}$. Fig. 9 shows the design of the air coil, and Table II shows its parameters.

Fig. 10 shows each layer of the prototype of an integrated air coil. The insulator of the PCBs is FR-4, and the copper width of the coil wire is set $0.5 \mathrm{~mm}$. Stray parameters of the prototype are measured, and the frequency characteristic of the current sensor is evaluated according to Step 5 . 
TABLE III

COMPARISON OF THE CURRENT SENSOR PARAMETERS

\begin{tabular}{l|l|l|l}
\hline Parameters & Theoretical & Simulation @ 1 MHz & Measurement \\
\hline \hline$M$ & $13.4 \mathrm{nH}$ & $13.3 \mathrm{nH}$ & $11.6 \mathrm{nH}$ \\
\hline$C_{\text {bus }}$ & $92.4 \mathrm{pF}$ & - & $100 \mathrm{pF}$ \\
\hline$L_{\text {bus }}$ & - & $4.10 \mathrm{nH}$ & $3.90 \mathrm{nH}$ \\
\hline$L_{\text {coil }}$ & - & $751 \mathrm{nH}$ & $840 \mathrm{nH}$ \\
\hline
\end{tabular}

TABLE IV

Current Sensor Parameters for the Equivalent Circuit

\begin{tabular}{l|l}
\hline Parameters & Measurement \\
\hline \hline$M$ & $11.6 \mathrm{nH}$ \\
\hline$C_{\text {bus }}$ & $100 \mathrm{pF}$ \\
\hline$L_{\text {coil }}^{\prime}$ & $2.10 \mathrm{nH}$ \\
\hline$R^{\prime}$ & $2.50 \Omega$ \\
\hline
\end{tabular}

\section{Prototype Evaluation}

To validate the design values obtained based on theory and through a simulation, the mutual inductance of the prototype is measured. A sinusoidal waveform is injected into the bus bars at $1 \mathrm{MHz}$ using a power amplifier (HSA4101, NF Corporation, Yokohama, Japan) for mutual inductance measurement. The other parameters of the prototype are measured using timedomain reflectometry (TDR). Table III shows the measurement results in comparison with theoretical or simulation results. As shown in Table III, all parameters demonstrate a good agreement with the theory or simulation. Besides, $C_{t-c}$ and $C_{b-c}$ are measured by TDR, and their values are 63.7 and $64.5 \mathrm{pF}$. It is confirmed that those capacitances are smaller than the bus-bar capacitance.

Table IV shows the value of the current sensor parameters for the equivalent circuit. The air coil self-inductance $L_{\text {coil }}$ is changed to $L_{\text {coil }}^{\prime}$ in a T-shaped equivalent circuit. In addition, $L_{\text {coil }}^{\prime}$ is obtained by considering the number of coil turns $N_{\text {coil }}$ and the measurement condition. The reason is that the bus-bar side is considered to the primary side, and the air coil side is considered to the secondary side when the parameters are assigned to the equivalent circuit. The value of $L_{\text {coil }}^{\prime}$ is smaller than the mutual inductance $M$. Therefore, the output resistance is set to $1 \mathrm{k} \Omega$ to satisfy (9) until $100 \mathrm{MHz}$.

\section{Frequency Characteristic of the Prototype}

An equivalent circuit with the measured parameters is employed to derive the frequency characteristic of the prototype. The gain is defined using (11). Fig. 11 shows the comparison of frequency characteristics between the characteristic from Fig. 5 (blue line), the characteristic from Fig. 6 (red dashed line), and the characteristic by using only the mutual inductance (black line). The characteristics from Fig. 5 and from Fig. 6 are similar until $100 \mathrm{MHz}$. Therefore, the equivalent circuit can be simplified, as shown in Fig. 6. Besides, it is confirmed that the difference between the characteristic from Fig. 6 and the characteristic by using only the mutual inductance appears from dozens of $\mathrm{MHz}$ in Fig. 11. Accordingly, the postprocessing method must achieve a bandwidth of $100 \mathrm{MHz}$ based on Fig. 6 .
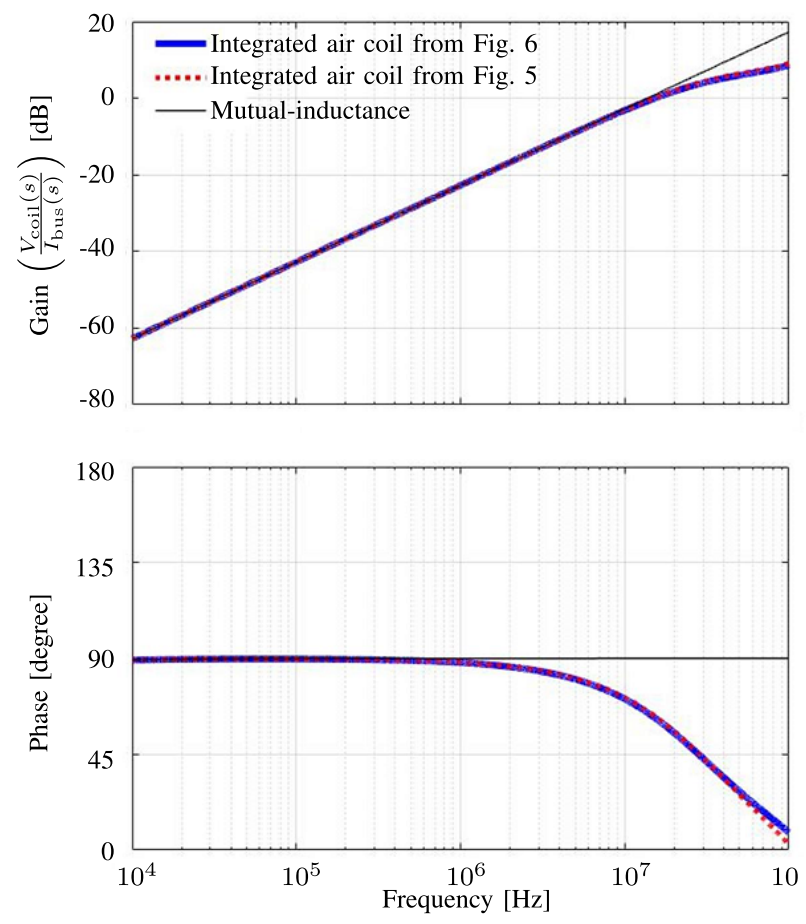

Fig. 11. Bode plot of the current sensor prototype of Figs. 5 and 6, and the bode plot of only with mutual inductance.

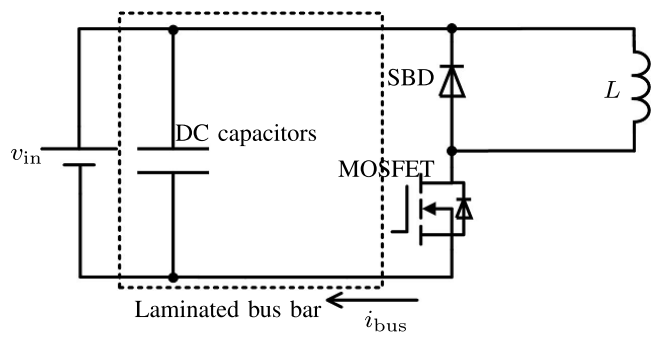

Fig. 12. Equivalent circuit of the buck converter for the double pulse test.

\section{E. Experimental Validation}

Fig. 12 shows the circuit diagram of a buck converter, and Fig. 13 shows a picture of a buck converter circuit. The red dashed line shows the location of the current sensor in Fig. 13. The experimental current is set to $10 \mathrm{~A}$. A Schottky barrier diode (SBD, CREE C3D10060A) and a MOSFET (SPP20N60S5 Infineon, Neubiberg, Germany) are used as the power devices in the buck chopper circuit.

The induced voltage $v_{\text {coil }}$ is the derivative waveform of the bus-bar current $i_{\text {bus }}$. The voltage $v_{\text {coil }}$ is measured using an oscilloscope (HD4096 LeCroy, Chestnut Ridge, NY, USA). The sampling rate is set to $2.5 \mathrm{GS} / \mathrm{s}$, and the vertical resolution is $12 \mathrm{~b}$. The bus-bar current using only the mutual inductance is calculated from (5), and the current using a postprocessing method is calculated from (11).

\section{F. Experiment Results}

Fig. 14 shows the induced voltage of the air coil $v_{\text {coil }}$ during a turnoff operation. One current waveform is calculated using (5), and the other is rebuilt through a postprocessing 


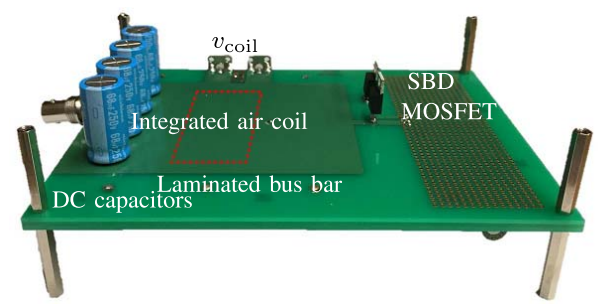

Fig. 13. Prototype of buck converter circuit.

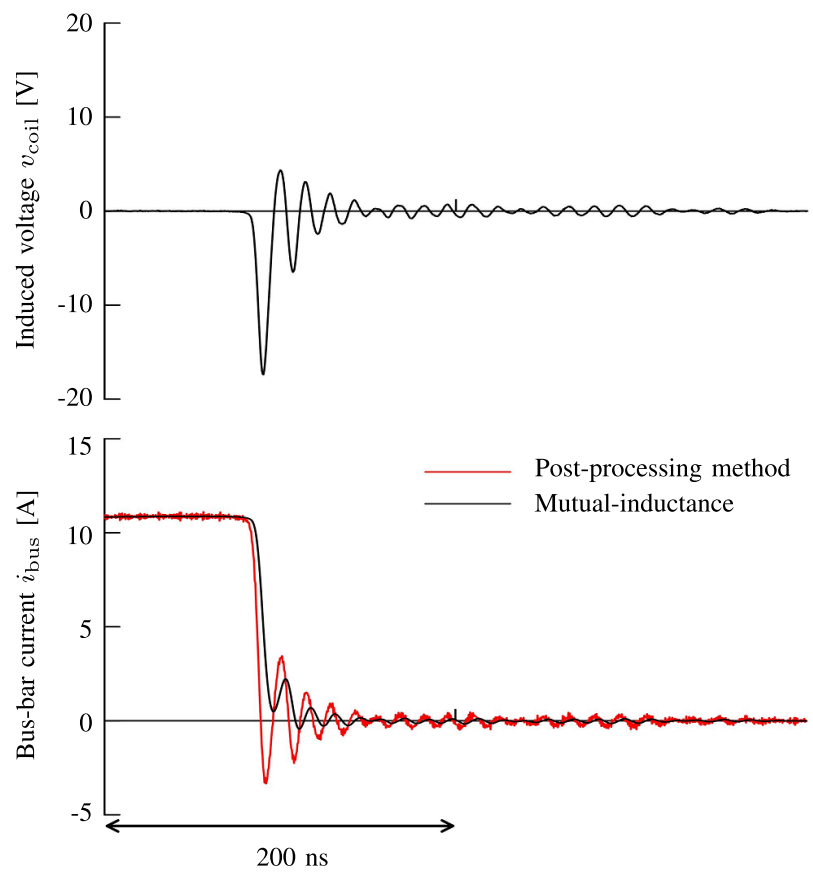

Fig. 14. Induced voltage of air coil and a comparison of the bus-bar current waveform during turn-OFF operation.

method (Fig. 14). The current waveforms are obtained through a numerical integration offline of the oscilloscope using $v_{\text {coil }}$. In turn-OFF operation, the dc offset is added to the waveform and the peak value of $v_{\text {coil }}$ determines the offset value. The absolute value of the maximum $d i_{\text {bus }} / d t$ is $1.22 \mathrm{~A} / \mathrm{ns}$. Therefore, it is confirmed that the voltage of $v_{\text {coil }}$ is less than $20 \mathrm{~V}$, which is set through the mutual inductance. The dc bias component has been reconstructed and added to both waveforms in Fig. 14. The frequency component is $71.4 \mathrm{MHz}$ for each of the current waveforms. Fig. 15 shows the voltage $v_{\text {coil }}$ and each bus-bar current waveforms during a turn-ON operation. The frequency component is $83.3 \mathrm{MHz}$ for each of the current waveforms.

From both operations, it is observed that the bus-bar current waveform obtained through only the mutual inductance (i.e., without postprocessing) fails to represent the frequency components at dozens MHz. A more accurate bus-bar current can be obtained through the postprocessing method, and the amplitude can be restored. As shown in Figs. 14 and 15, a current sensor using the postprocessing method can accurately measure a switching waveform containing dozens of $\mathrm{MHz}$ frequency components.

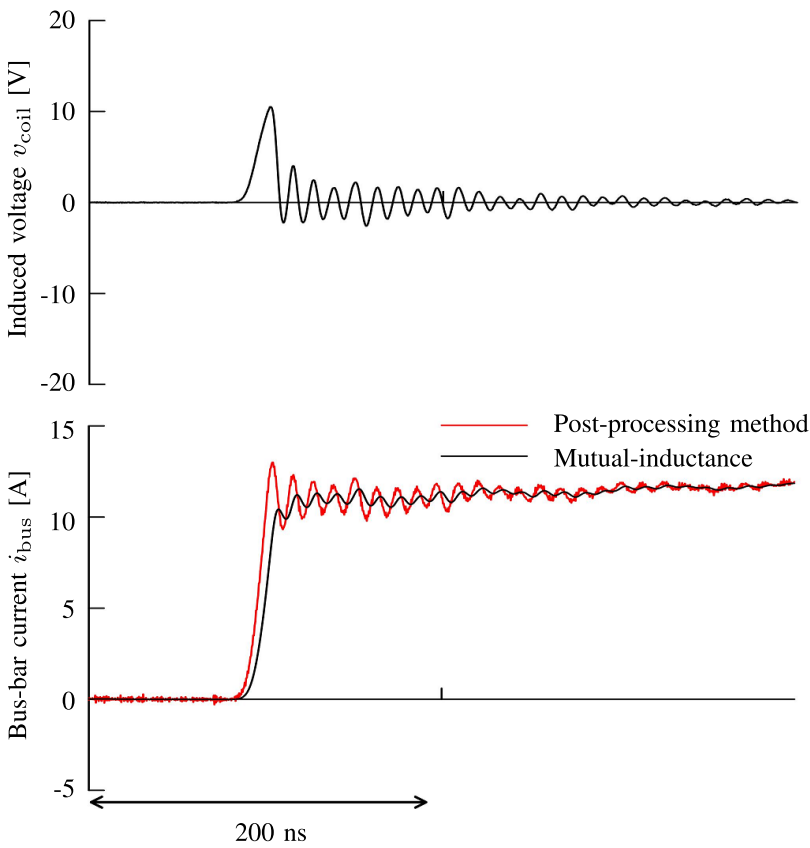

Fig. 15. Induced voltage of air coil and a comparison of the bus-bar current waveform during turn-ON operation.

Besides, the measurement accuracy of the integrated air coil depends on the oscilloscope because the processing method takes $v_{\text {coil }}$ to rebuilt measured current offline. Therefore, the measurement accuracy is less than $0.1 \%$ at the maximum induced voltage in this experiment with this measurement system.

\section{CONCLUSION}

This article proposed the design procedure of an integrated air coil in a laminated bus bar. The structure of the current sensor and the frequency characteristic of the current sensor ware discussed. The design procedure was then summarized, and the procedure showed that the current sensor could be designed under the limitation of the laminated bus bar. A postprocessing method used to achieve a frequency bandwidth of $100 \mathrm{MHz}$ was proposed. The validity of the design procedure and the postprocessing method were confirmed using the prototype through a double pulse test under a 10-A current.

\section{REFERENCES}

[1] K. Yamaguchi, K. Katsura, T. Yamada, and Y. Sato, "Comprehensive study on gate driver for SiC-MOSFETs with gate boost," IEEJ J. Ind. Appl., vol. 7, no. 3, pp. 218-228, May 2018.

[2] H.-C. Yang, R. Simanjorang, and K. Y. See, "A method of junction temperature estimation for $\mathrm{SiC}$ power MOSFETs via turn-on saturation current measurement," IEEJ J. Ind. Appl., vol. 8, no. 2, pp. 306-313, Mar. 2019.

[3] J. M. Allocco, "Laminated bus bars for power system interconnects," in Proc. IEEE APEC, 1997, vol. 2, pp. 585-589.

[4] M. C. Caponet, F. Profumo, R. W. De Doncker, and A. Tenconi, "Low stray inductance bus bar design and construction for good EMC performance in power electronic circuits," IEEE Trans. Power Electron. vol. 17, no. 2, pp. 225-231, Mar. 2002.

[5] C. Chen, X. Pei, Y. Chen, and Y. Kang, "Investigation, evaluation, and optimization of stray inductance in laminated busbar," IEEE Trans. Power Electron., vol. 29, no. 7, pp. 3679-3693, Jul. 2014. 
[6] M. Ando and K. Wada, "Design of acceptable stray inductance based on scaling method for power electronics circuits," IEEE J. Emerg. Sel. Topics Power Electron., vol. 5, no. 1, pp. 568-575, Mar. 2017.

[7] A. D. Callegaro et al., "Bus bar design for high-power inverters," IEEE Trans. Power Electron., vol. 33, no. 3, pp. 2354-2367, Mar. 2018.

[8] K. Sun, J. Wang, R. Burgos, D. Boroyevich, Y. Kang, and E. Choi, "Analysis and design of an overcurrent protection scheme based on parasitic inductance of SiC MOSFET power module," in Proc. IEEE Appl. Power Electron. Conf. Exposit. (APEC), Mar. 2018, pp. 2806-2812.

[9] Z. Zhang, B. Guo, F. F. Wang, E. A. Jones, L. M. Tolbert, and B. J. Blalock, "Methodology for wide band-gap device dynamic characterization," IEEE Trans. Power Electron., vol. 32, no. 12, pp. 9307-9318, Dec. 2017.

[10] J. Wang, Z. Shen, R. Burgos, and D. Boroyevich, "Integrated switch current sensor for shortcircuit protection and current control of $1.7-\mathrm{kV}$ SiC MOSFET modules," in Proc. IEEE Energy Convers. Congr. Exposit. (ECCE), Sep. 2016, pp. 1-7.

[11] Y. Ren, M. Xu, J. Zhou, and F. C. Lee, "Analytical loss model of power MOSFET," IEEE Trans. Power Electron., vol. 21, no. 2, pp. 310-319, Mar. 2006.

[12] A. Hino and K. Wada, "Resonance analysis focusing on stray inductance and capacitance of laminated bus bars," IEEJ J. Ind. Appl., vol. 5, no. 6, pp. 407-412, 2016.

[13] G. Engelmann, A. Sewergin, M. Neubert, and R. W. De Doncker, "Design challenges of $\mathrm{SiC}$ devices for Low- and medium-voltage DC-DC converters," in Proc. Int. Power Electron. Conf. (IPEC-NiigataECCE Asia), May 2018, pp. 505-511.

[14] S. Ziegler, R. C. Woodward, H. H.-C. Iu, and L. J. Borle, "Current sensing techniques: A review," IEEE Sensors J., vol. 9, no. 4, pp. 354-376, Apr. 2009.

[15] W. Kim, S. Luo, G.-Q. Lu, and K. D. T. Ngo, "Integrated current sensor using giant magneto resistive (GMR) field detector for planar power module," in Proc. 28th Annu. IEEE Appl. Power Electron. Conf. Expo. (APEC), Mar. 2013, pp. 2498-2505.

[16] J. Wang et al., "Infinity sensor: Temperature sensing in $\mathrm{GaN}$ power devices using peak di/dt," in Proc. IEEE Energy Convers. Congr. Expo. (ECCE), Sep. 2018, pp. 884-890.

[17] M. Adelmund, C. Bodeker, and N. Kaminski, "Optimisation of shunt resistors for fast transients," in Proc. PCIM, 2016, pp. 1-8.

[18] S. Iyasu, Y. Hayashi, Y. Handa, K. Nakamura, and K. Wada, "A bidirectional current sensor based on CT with diode rectifier and MOSFET for bidirectional current-fed DC-DC converter," IEEJ J. Ind. Appl., vol. 8, no. 3, pp. 437-443, May 2019.

[19] Z. Yan and L. Hongbin, "The reliable design of PCB rogowski coil current transformer," in Proc. Int. Conf. Power Syst. Technol., Oct. 2006, pp. $1-4$.

[20] High Accuracy AC Current Measurement Reference Design Using PCB Rogowski Coil Sensor, T. Instruments, Lymington, U.K., Jul. 2016.

[21] L. Zhao, J. D. van Wyk, and W. G. Odendaal, "Planar embedded pickup coil sensor for integrated power electronic modules," in Proc. APEC, 2004, pp. 945-951.

[22] Y. Xue, J. Lu, Z. Wang, L. M. Tolbert, B. J. Blalock, and F. Wang, "A compact planar rogowski coil current sensor for active current balancing of parallel-connected silicon carbide MOSFETs," in Proc. IEEE Energy Convers. Congr. Expo. (ECCE), Sep. 2014, pp. 4685-4690.

[23] K. Hasegawa, S. Takahara, S. Tabata, M. Tsukuda, and I. Omura, "A new output current measurement method with tiny PCB sensors capable of being embedded in an IGBT module," IEEE Trans. Power Electron., vol. 32, no. 3, pp. 1707-1712, Mar. 2017.

[24] Y. Kuwabara, K. Wada, J.-M. Guichon, J.-L. Schanen, and J. Roudet, "Implementation and performance of a current sensor for a laminated bus bar," IEEE Trans. Ind. Appl., vol. 54, no. 3, pp. 2579-2587, May 2018.

[25] Y. Kuwabara, K. Wada, J.-M. Guichon, J.-L. Schanen, and J. Roudet, "Bus bar embedded rogowski coil," in Proc. IEEE Appl. Power Electron. Conf. Expo. (APEC), Mar. 2018, pp. 2821-2826.

[26] R. J. Pasterczyk, C. Martin, J.-M. Guichon, and J.-L. Schanen, "Planar busbar optimization regarding current sharing and stray inductance minimization," in Proc. Eur. Conf. Power Electron. Appl., Sep. 2005 , p. 9.

[27] S. Mocevic et al., "Phase current reconstruction based on rogowski coils integrated on gate driver of SiC MOSFET half-bridge module for continuous and discontinuous PWM inverter applications," in Proc. IEEE Appl. Power Electron. Conf. Expo. (APEC), Mar. 2019, pp. 1029-1036.

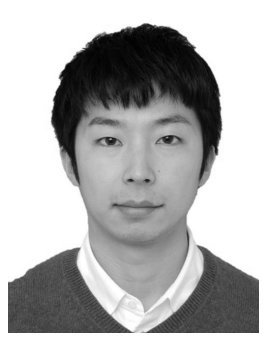

Yoshikazu Kuwabara (Student Member, IEEE) was born in Kanagawa, Japan, in 1991. He received the B.S., M.S. and Ph.D. degrees in electrical engineering from Tokyo Metropolitan University, Tokyo, Japan, in 2015, 2017, and 2020, respectively.

His current research interest includes the current sensor for integrated technologies in power electronics circuits.

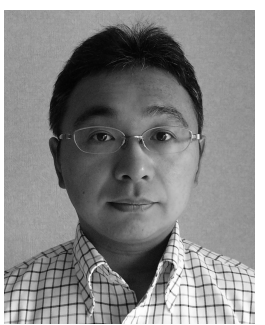

Keiji Wada (Senior Member, IEEE) was born in Hokkaido, Japan. He received the Ph.D. degree in electrical engineering from Okayama University, Okayama, Japan, in 2000.

From 2000 to 2006, he was a Research Associate with Tokyo Metropolitan University, Tokyo, Japan, and the Tokyo Institute of Technology, Tokyo. Since 2006, he has been an Associate Professor with Tokyo Metropolitan University. His current research interests include the medium-voltage inverter, electromagnetic interference filters, and active power filters.

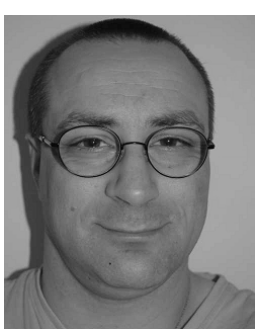

Jean-Michel Guichon received the Diploma degree in electrical engineering and the Ph.D. degree from the Grenoble Institute of Technology, Grenoble, France, in 1998 and 2001, respectively.

$\mathrm{He}$ is currently an Associate Professor with the University Grenoble Alpes, Grenoble. Since 2003, he has been with the Grenoble Electrical Engineering Laboratory, working in the field of electromagnetic modelization for power electronics. He is focused on the development of numerical tools to compute the electromagnetic field and the electrical behavior in low frequency (without propagation).

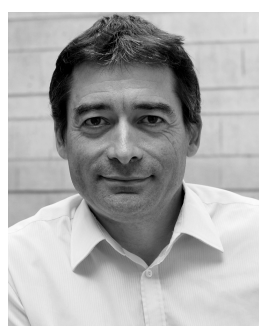

Jean-Luc Schanen (Senior Member, IEEE) received the Ph.D. degree from Grenoble Institute of Technology, Grenoble, France, in 1990 and 1994, respectively.

Since 2003, he has been a Professor with the Universite Grenoble Alpes, Grenoble, where he is currently leading the power electronics research group of the G2ELab. He is also the Deputy Director with the Engineering School Energy, Water and Environment, Grenoble. His research activities are focused on the technological design of power electronics systems, including electromagnetic compatibility (EMC) aspects. His group develops models and tools for power converters optimization.

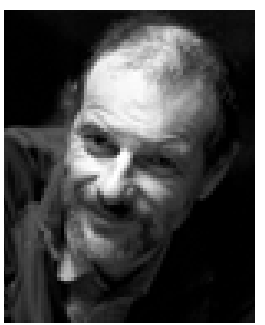

James Roudet was born in Grenoble, France, in 1963. He received the Diploma degree in electrical engineering and the Ph.D. degree from Grenoble University, Grenoble, in 1986 and 1990, respectively.

$\mathrm{He}$ is currently a Professor of power electronics with the Grenoble Electrical Engineering Laboratory (G2ELab), Grenoble University (Universite Joseph Fourier, France). The first research interest concerned resonant converters. He promoted electromagnetic compatibility (EMC) activities in the field of power electronics and developed a leading activity in the technological design of power converters. He is also the Director of G2ELab, after several years of leading the power electronics team. 